\title{
Beyond the Limit of Deep X-ray Surveys: Galaxies or AGN?
}

\author{
Richard E. Griffiths ${ }^{1}$, Takamitsu Miyaji ${ }^{1}$, Adam Knudson ${ }^{1}$ and \\ Matthew Schurch \\ ${ }^{1}$ Department of Physics, Carnegie Mellon University, Pittsburgh, PA 15213, USA \\ email: griffith@astro.phys.cmu.edu
}

\begin{abstract}
The great sensitivities of the Chandra X-ray Observatory and XMM-Newton are allowing us to explore the X-ray emission from galaxies at moderate to high redshift. By using the stacking method with CXO data, we show that we can detect the ensemble emission from normal elliptical, spiral and irregular galaxies out to redshifts approaching one. The average Xray luminosity can then be compared with the results of models of the evolution in the numbers of X-ray binaries and can possibly be used to constrain models of star formation. In order to account for the increasing luminosity of spiral galaxies from low to moderate redshift, AGN components may need to be invoked.
\end{abstract}

Keywords. X-ray background, galaxies, number counts, X-ray binaries, AGN.

\section{Introduction}

Deep surveys in X-ray astronomy had the initial goal of solving the problem of the origin of the extragalactic X-ray background, and these surveys have now shown that the $\mathrm{XRB}$ is largely comprised of the evolving populations of AGN, some heavily absorbed (Hasinger et al. 2005). But the deep surveys with the Chandra X-ray Observatory (CXO) have shown that normal galaxies are also detected. The initial 1Ms survey of the Hubble Deep Field (HDF) North demonstrated that, at flux levels approaching $10^{-16} \mathrm{cgs}(0.5-$ $2.0 \mathrm{keV}$ ), about a third of the X-ray sources were identified with galaxies (Horneschmeier et al. 2002, 2003). The extension of this survey to 2Ms. has confirmed and expanded these findings.

We can thus begin to explore the evolution of extragalactic source populations in addition to the AGN, LINERs and clusters of galaxies which dominated the number counts in the flux ranges explored by the Einstein, ROSAT and ASCA observatories in the previous two decades. It has long been recognised that normal galaxy populations would eventually be observed in deep X-ray surveys given enough sensitivity. It was not clear that this would happen with the Chandra X-ray Observatory.

\section{Deep Surveys and Source Counts}

The number counts in the HDF-N have been measured by Miyaji \& Griffiths (2002), and extended to fluxes below $10^{-17} \mathrm{ergs} \mathrm{cm}^{-2} \mathrm{~s}^{-1}$ in the soft band $(0.5-2 \mathrm{keV})$ and to $10^{-16}$ ergs $\mathrm{cm}^{-2} \mathrm{~s}^{-1}$ in the hard band $(2-10 \mathrm{keV})$ by analysis of the fluctuations which remain after removal of the individual discrete source detections. Below this limit, the fluctuation analysis shows that the number counts continue to rise, as shown in Fig. 1, with a slope consistent with that between $10^{-15}$ and $10^{-16} \mathrm{ergs}^{-2} \mathrm{~s}^{-1}$.

At X-ray fluxes between $10^{-15}$ and $10^{-16} \mathrm{ergs} \mathrm{cm}^{-2} \mathrm{~s}^{-1}$ in the HDF-N, Hornschemeier et al. $(2002,2003)$ showed that about a third of sources were identified with galaxies. 


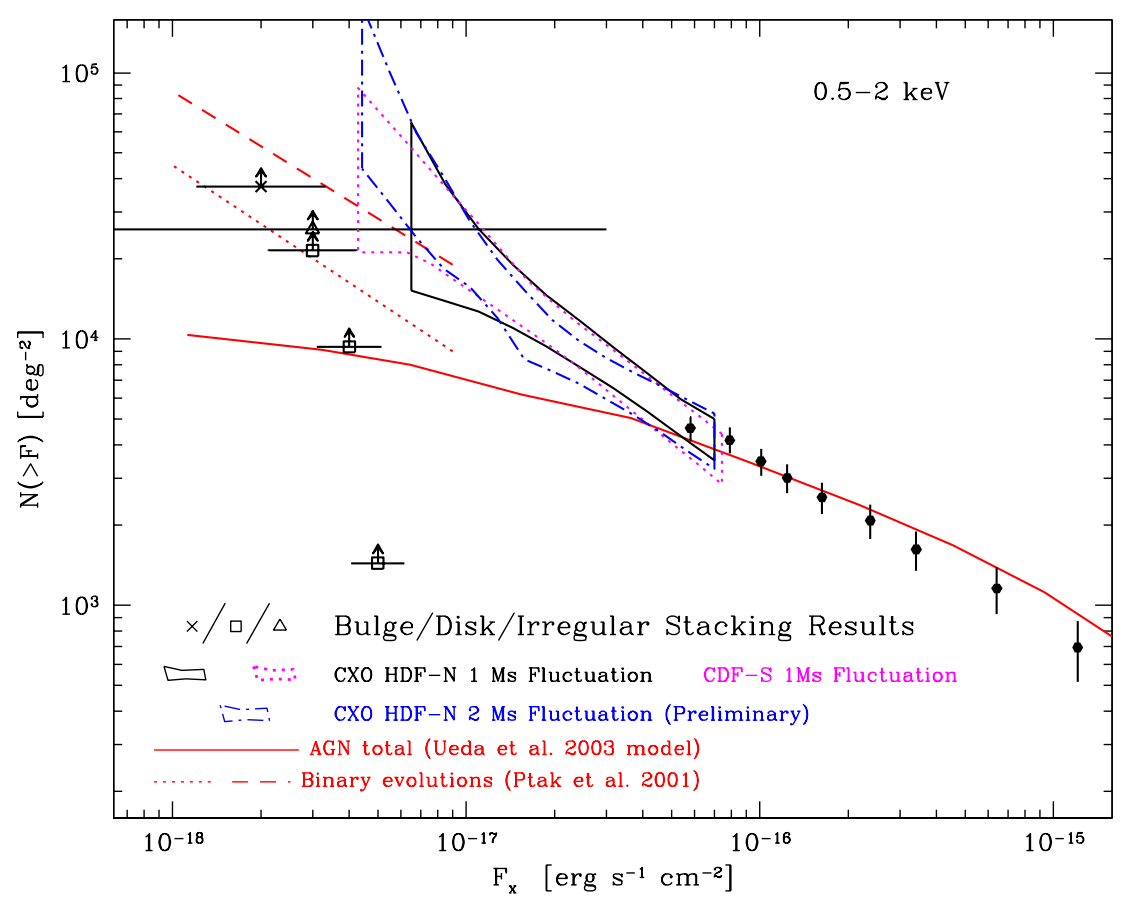

Figure 1. X-ray Number Counts from the Hubble/Chandra Deep Field North. The 1 Ms "fish-tail" fluctuation limits are from Miyaji \& Griffiths (2002), and similar results were found from the HDF-S. Fluctuation limits from the 2Ms CDF-N are preliminary and possibly contaminated by cosmic-ray background events. Points showing stacked galaxies of different morphological types are all lower limits - stacking was done on optical galaxies in the HDF-N and the data points represent the average fluxes of the stacked galaxies. For any given morphological type, it is unlikely, however, that the number counts due to them are much higher than those shown here, unless contaminated by AGN. The prediction of AGN number counts is from Ueda et al. (2003). An estimate of the number counts from X-ray binary populations in normal galaxies was made by Ptak et al. (2001).

The extension of this survey to $2 \mathrm{Ms}$. has confirmed and expanded these findings. We infer that the number counts in the region explored with fluctuations (the boxed area in Fig. 1) are unlikely to be due to AGN. Furthermore, the current best models for the AGN contributions to the number counts fall well below the fluctuations. The fluctuations analysis shows that the number counts are approximately 20,000 - 40,000 per sq. deg. at X-ray fluxes of $10^{-17} \mathrm{ergs} \mathrm{cm}^{-2} \mathrm{~s}^{-1}$. Such number counts match those of the optical counts of galaxies at $\mathrm{B}=24$. We therefore explore the possibility of X-ray detection of faint galaxies in the HDF-N by using the stacking method.

\section{Selection of Galaxies by Morphological Type and Luminosity}

During the execution of the Medium Deep Survey using the Hubble Space Telescope, software was developed for the automated classification of galaxies into spirals (exponential disks), ellipticals ('de Vaucouleurs' profiles) and irregular galaxies which exhibited large residual images after the removal of disk or bulge profiles (Ratnatunga, Griffiths \& Ostrander 1999). This survey showed, for example, that the fraction of irregulars rises from $12 \%$ locally to $30 \%$ at a redshift of 0.5 . 


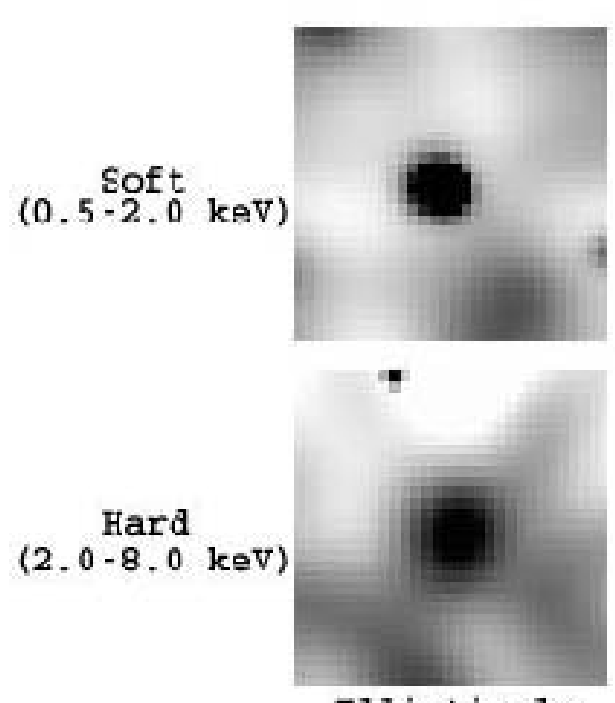

Ellipticals

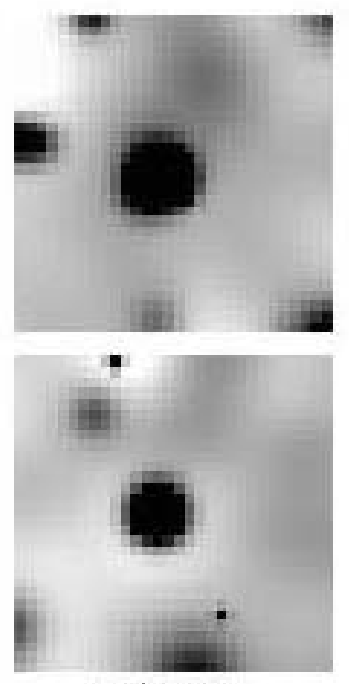

Epirala

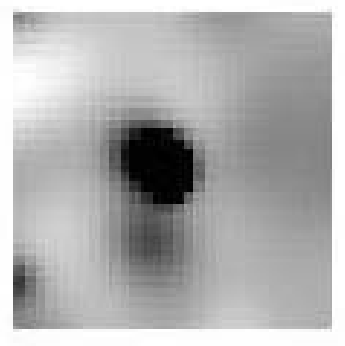

No

Detection

Irregulare

Figure 2. Stacked X-ray images of ensembles of galaxies.

We have applied this MDS software and analysis to the HST images of the HDF-N and other deep HST surveys. In those fields where we have deep CXO observations, we can then examine the X-ray images for the presence of X-rays from the galaxies of differing broad morphological type.

\section{Results of 'Stacking'}

Although the fluctuations analysis gives us an indication of the number counts of X-ray sources at the faintest flux levels currently accessible, they do not give us any indication of the nature of the sources contributing to the fluctuations. How do we find out the possible nature of these sources? One method is that of 'stacking', i.e. the summation of sub-images centered on objects selected at another wavelength. Brandt et al. (2001) and Horneschmeier et al. (2002) have used this method on early CXO data of the HDF-N. In the HDF, we have the advantage of being able to use the HST images themselves to select various types of galaxies for the stacking process, using the software developed as part of the HST Medium Deep Survey (Ratnatunga, Griffiths and Ostrander 1999). We have now done this for elliptical, spiral and irregular galaxies, and some of the results are shown in Figure 2.

As the figure shows, the spiral and elliptical galaxies are detected at high confidence in both the soft and hard energy bands, but the irregular galaxies are detected in the soft band only. The median redshifts are 0.87 for the 27 ellipticals, 0.49 for the 54 spirals and 1.55 for the 57 irregulars in these stacked images. Monte Carlo simulations have been used to verify the statistical confidence in these results.

The median X-ray luminosities $(0.5-2 \mathrm{keV})$ are $5 \times 10^{39} \mathrm{ergs} \mathrm{s}^{-1}$ for the ellipticals, $6 \times 10^{39} \mathrm{ergs} \mathrm{s}^{-1}$ for the spirals and $2 \times 10^{40} \mathrm{ergs} \mathrm{s}^{-1}$ for the irregulars, consistent with 
their B-band luminosities and the average values for $L_{X} / L_{B}$ for the galaxy types. Typical galaxy fluxes are $\sim 2-4 \times 10^{-18} \mathrm{ergs}^{-2} \mathrm{~s}^{-1}$.

\section{X-ray Evolution of Galaxies}

The observed X-ray evolution of spiral galaxies out to $\mathrm{z}=0.7$ is observed to be in excess of the expected value based on the evolution of binary X-ray populations. The observed evolution is better matched with a population of AGN such that the AGN luminosity is 0.1 of the galaxy luminosity in $30 \%$ of the galaxies at $\mathrm{z}=0.7$.

There are several problems which need to be solved or investigated in support of the interpretation of these results: (i) the evolution of low-mass X-ray binaries (LMXRB), (ii) the evolution of high-mass X-ray binaries (HMXRB), (iii) the evolution in the number of ultraluminous X-ray (ULX) objects and (iv) SNR and hot gas components.

\section{Conclusions}

Results from the stacking analysis of normal galaxy populations applied to the CXO deep survey of the HDF-N show that normal galaxy populations are observable in these stacks out to redshifts of $\sim 1$. The average X-ray fluxes observed in these stacks are consistent with the numbers and fluxes inferred from the fluctuation analysis of the CXO data. We conclude that the fluctuations are therefore caused primarily by normal galaxy populations and that such deep X-ray surveys will eventually allow us to constrain the evolution of the binary source populations within these galaxies, using the relationship between HMXB numbers and the SFR of nearby galaxies. We have tentative evidence for the presence of AGN in some fraction of normal spiral agalxies at moderate redshift.

\section{Acknowledgements}

We acknowledge support from NASA grants NAG5-9902, NAG5-10875 and subcontract 2247-CMU-NASA-1128 from PSU (under NAS8-00128).

\section{References}

Anderson, S. \& Margon, B. 1987, Astrophys. J. 314, 111

Brandt, W. N., Hornschemeier, A. E., Schneider, D. P., Alexander, D. M., Bauer, F. E., Garmire, G., \& Vignali, C. 2001, Astrophys. J. 558, L5

Ghosh, K. \& White, N. 2001, Astrophys. J. 559, L97

Hasinger, G., et al. 2005, Astron. 8 Astrophys. in press

Hornschemeier, A. E., Brandt, W. N., Alexander, D. M., Bauer, F. E., Garmire, G. P., Schneider, D. P., Bautz, M. W., \& Chartas, G. 2002, Astrophys. J. 568, 82

Hornschemeier, A. E. and the CDF-N team 2003, X-rays at Sharp Focus: ASP Conf. Series, eds. S. Vrtilek, E. M. Schlegel, L. Kuhi

Miyaji, T. \& Griffiths, R.E. 2002, Astrophys. J. 564, L5

Norman, C., et al. 2004, Astrophys. J. 607721

Ptak, A. \& Griffiths, R. E. 2001, Astrophys. J. 559, L91

Ratnatunga, K., Griffiths, R. E., \& Ostrander, E. J. 1999, Astron. J. 118, 86

Ueda, Y., Akiyama, M., Ohta, K., \& Miyaji, T. 2003, Astrophys. J. 598, 886 